\title{
Think fast! The implications of emphasizing urgency in decision-making
}

\author{
Nathan J. Evans \\ Department of Psychology, University of Amsterdam, The Netherlands
}

Word count: 9,945

Keywords:

Decision-making — Speed-accuracy tradeoff — Evidence accumulation models — Selective influence

Correspondence concerning this article may be addressed to: Nathan Evans (nathan.j.evans@uon.edu.au) 


\begin{abstract}
Evidence accumulation models (EAMs) have become the dominant explanation of how the decision-making process operates, proposing that decisions are the result of a process of evidence accumulation. The primary use of EAMs has been as "measurement tools" of the underlying decision-making process, where researchers apply EAMs to empirical data to estimate participants' task ability (i.e., the "drift rate"), response caution (i.e., the "decision threshold"), and the time taken for other processes (i.e., the "non-decision time"), making EAMs a powerful tool for discriminating between competing psychological theories. Recent studies have brought into question the mapping between the latent parameters of EAMs and the theoretical constructs that they are thought to represent, showing that emphasizing urgent responding - which intuitively should selectively influence decision threshold - may also influence drift rate and/or non-decision time. However, these findings have been mixed, leading to differences in opinion between experts in the field. The current study aims to provide a more conclusive answer to the implications of emphasizing urgent responding, providing a re-analyse of 6 data sets from previous studies using two different EAMs - the diffusion model and the linear ballistic accumulator (LBA) - with state-of-the-art methods for model selection based inference. The findings display clear evidence for a difference in conclusions between the two models, with the diffusion model suggesting that decision threshold and non-decision time decrease when urgency is emphasized, and the LBA suggesting that decision threshold and drift rate decrease when urgency is emphasized. Furthermore, although these models disagree regarding whether non-decision time or drift rate decrease under urgency emphasis, both show clear evidence that emphasizing urgency does not selectively influence decision threshold. These findings suggest that researchers should revise their assumptions about certain experimental manipulations, the specification of certain EAMs, or perhaps both.
\end{abstract}




\section{Introduction}

Decision-making has been one of the most widely studied aspects of human cognition, being a fundamental topic of research in psychology, neuroscience, and economics. Evidence accumulation models (EAMs; Stone, 1960; see Donkin, Averell, Brown, \& Heathcote, 2009 for an introduction and Evans \& Wagenmakers, 2020 for a recent review) have become one of the most dominant explanations of how the decision-making process operates, proposing that decisions are the result of a process of evidence accumulation, where the evidence gradually accumulates for each possible decision alternative over time at some rate (known as the "drift rate"), until the evidence for one alternative reaches a level that triggers a response (known as the "decision threshold"). Importantly, EAMs are able to assess response choice and response time in unison, meaning that these models can account for commonly observed tradeoffs between response speed and accuracy (i.e., performing a task more carefully to increase accuracy), and decompose response choice and response time for each trial into the latent variables of the decision-making process, such as drift rate, decision threshold, and the time taken for processes that are not directly part of the decision (known as the "non-decision time").

The primary use of EAMs has been as "measurement tools" of the underlying decision-making process, where researchers apply EAMs to empirical data to estimate the drift rate, decision threshold, and non-decision time of each participant in each experimental condition (i.e., model application under the categorization of Crüwell, Stefan, \& Evans, 2019). Researchers then assess whether these estimated parameters differ between experimental conditions or groups of participants, allowing researchers to make direct inferences about the components of the underlying decision-making process, rather than indirect inferences using the differences in observed variables (e.g., response choice and response time). Importantly, when the predictions of theories can be conceptualized as differences in these 
latent parameters over conditions or groups, EAMs can be a powerful tool for discriminating between competing psychological theories. For example, a well-established finding within the ageing literature is that older adults have slower response times than younger adults in a range of cognitive tasks. Researchers had postulated that this difference was due to a cognitive slowdown (Salthouse, 1996), where older adults experience cognitive decline, and therefore, are slower at cognitive tasks than their younger counterparts. However, the assessment of response time alone was not sufficient to distinguish between this explanation and other alternatives; for example, older adults could instead be more cautious, or slower at motor movements, than younger adults. Using the diffusion model (Ratcliff, 1978) - the most commonly applied EAM - Ratcliff, Thapar, and McKoon (2001) was able to contrast these different theoretical accounts: a change in drift rate between groups would reflect a cognitive slowdown, a change in decision threshold would reflect an increase in caution, and a change in non-decision time would reflect slower motor responding. Importantly, Ratcliff et al. (2001) found that older adults only differed from younger adults in the decision threshold and non-decision time parameters, finding evidence for the increased caution explanation over the cognitive slowdown explanation. Similar questions have been answered in a range of other fields, such as performance optimality (Bogacz, Brown, Moehlis, Holmes, \& Cohen, 2006; Starns \& Ratcliff, 2012; Evans, Bennett, \& Brown, 2019), genetics (Evans, Steyvers, \& Brown, 2018), executive control (Hübner, Steinhauser, \& Lehle, 2010; White, Ratcliff, \& Starns, 2011; Ulrich, Schröter, Leuthold, \& Birngruber, 2015; Evans \& Servant, 2020), driver distraction (Tillman, Strayer, Eidels, \& Heathcote, 2017), ego depletion (Lin, Saunders, Friese, Evans, \& Inzlicht, 2020), and even the effects of inebriation (van Ravenzwaaij, Dutilh, \& Wagenmakers, 2012).

One fundamental assumption underlying EAMs and their use as measurement tools for answering theoretical questions is that each latent parameter maps onto a different 
theoretical construct. Specifically, the drift rate parameter is thought to map into how easy the task is for a specific person, being some combination of the person's ability at these specific types of tasks and how difficult the current task is overall, with easier tasks resulting in higher drift rates. Decision threshold is thought to map onto a person's decision strategy regarding their speed-accuracy tradeoff, where participants can either raise their threshold to respond more slowly with greater accuracy, or lower their threshold to respond more quickly with lesser accuracy. Non-decision time is thought to map onto how quickly perceptual processes feed into the decision process, and how quickly motor processes operate after a decision is made, with slower perceptual processing and/or slower motor movement resulting in longer non-decision times. Researchers also often use experimental manipulations as a means of influencing these different constructs (Dutilh et al., 2019). For example, researchers often present participants with different stimuli within a task, some of which are easier and some of which are harder. This manipulation is designed to influence the ease of the task, and only the ease of the task, and therefore, influence drift rate, and only drift rate (Ratcliff, 1978; Ratcliff \& Rouder, 1998; Evans, Hawkins, \& Brown, 2020). Different manipulations also exist for the other parameters, where researchers attempt to influence non-decision time by making motor responding quicker or more laborious (Lerche \& Voss, 2017), and attempt to influence decision threshold by instructing participants to pay more attention to speed or accuracy (Voss, Rothermund, \& Voss, 2004; Rae, Heathcote, Donkin, Averell, \& Brown, 2014; Lerche \& Voss, 2017; Dutilh et al., 2019). The assumption that a specific manipulation can influence a specific parameter, and only that specific parameter, is known as the "selective influence assumption", and some of these benchmark manipulations are key to our understanding of EAMs and the decision process.

One recent topic of discussion within the decision-making literature has been the accuracy of these selective influence assumptions. Specifically, although we assume that a 
specific manipulation should only influence a specific parameter, is this actually a sensible assumption to make in all cases? Or could some of these manipulations also unwittingly influence a second (or third) parameter? For selective influence manipulations of drift rate and non-decision time, this assumption seems intuitively sensible. Manipulations of drift rate typically involve using stimuli of varying difficulties within a single block of trials (though also see Starns \& Ratcliff, 2012), meaning that to systematically alter their decision threshold, participants would need to attempt to both 1) decide on whether the decision was easy or difficult before making the actual task decision, and 2) adjust their decision threshold mid-trial based on this difficulty decision (see Evans, Hawkins, \& Brown, 2020 for a test of the latter assumption). Furthermore, manipulations of the decision-making process (i.e., drift rate and decision threshold) should be distinct from manipulations of the motor process (i.e. non-decision time), meaning that changes in item difficulty should not influence non-decision time, and manipulations of motor responding should not influence drift rate or decision threshold (Lerche \& Voss, 2017). Most importantly, previous research has suggested the robustness of selective influence manipulations of drift rate and nondecision time (Voss et al., 2004; Lerche \& Voss, 2017; Dutilh et al., 2019), although it should be noted that there does not appear to be any universal agreement on a selective influence manipulation for non-decision time (Dutilh et al., 2019).

However, the selectivity of manipulations of decision threshold seem more questionable. Manipulations of decision threshold involve attempting to force participants to change their decision strategy to be either more urgent or more cautious, which is typically done through instructing participants to focus on either speed or accuracy in different experimental blocks (Dutilh, Forstmann, Vandekerckhove, \& Wagenmakers, 2013; Rae et al., 2014; Evans, Rae, Bushmakin, Rubin, \& Brown, 2017; Dutilh et al., 2019), or implementing deadlines in certain blocks of the task to force participants to be more urgent (Evans, 
Hawkins, \& Brown, 2020). However, it seems plausible that these manipulations could have unintended consequences on other components of the decision-making process. For example, telling participants to respond more quickly, or putting them under explicit deadlines, may put them under some amount of decision pressure or stress, which could decrease their task ability (Rae et al., 2014). An emphasis on urgency may also encourage participants to perform all parts of the process as fast as possible, meaning that they may execute motor responses faster than usual (Lerche \& Voss, 2017).

Previous research has suggested that encouraging urgency may result in more than just a decrease in decision threshold, finding that these manipulations can influence drift rate (Rae et al., 2014), non-decision time (Voss et al., 2004; Lerche \& Voss, 2017), or in some cases even result in participants adopting thresholds that decrease over time (Evans, Hawkins, \& Brown, 2020). However, previous findings have differed in how the selective influence assumption fails, and opinions are split between experts in the field. For example, in the recent many-labs project of Dutilh et al. (2019), researchers were required to identify which EAM parameters varied between experimental conditions in 14 different blinded empirical pseudo-experiments ${ }^{1}$. For pseudo-experiments that manipulated urgency emphasis through speed and accuracy instructions, the organizers assumed that this manipulation should selectively influence decision threshold, and therefore, that labs who identified only a change in decision threshold made the correct judgment. However, two alternative scoring rules were suggested by other researchers based on previous findings: that speed instructions should 1) decrease both threshold and drift rate, based on the conclusions of Rae et al. $(2014)^{2}$, or 2) decrease both threshold and non-decision time, based on the conclusions

\footnotetext{
${ }^{1}$ These are referred to as "pseudo-experiments" as all experiments were technically part of a single experiment with a large number of conditions, though labelled as different experiments for the purpose of the project.

${ }^{2}$ It should be noted that Rae et al. (2014) found evidence that speed instructions decrease threshold, drift rate, and non-decision time. However, the primary conclusion of Rae et al. (2014) was that speed instructions decrease both decrease threshold and drift rate, which is reflected in Dutilh et al. (2019)'s
} 
of Voss et al. (2004). These three different expert opinions on what urgency emphasis should influence resulted in three different scoring rules for the project, and each scoring rule resulted in a different lab being judged to have the best performance in the project. Importantly, these discrepancies show that expert opinions currently diverge on what components of the decision-making process are influenced by emphasizing urgent responding, and that these differences in opinion have meaningful consequences.

The diverging opinions of experts on what components of the decision-making process are influenced by emphasizing urgent responding appears to be the result of the diverging conclusions of previous studies that have investigated speed and accuracy emphasis instructions (Dutilh et al., 2019). However, it is unclear why these studies differed in their conclusions. One reason may be the varying methodologies employed by previous studies (see Evans, 2019 for a discussion), which have differed in factors such as the experimental design (i.e., the task), the choice of EAM for the application (i.e., the diffusion model or the linear ballistic accumulator), the analysis framework (i.e., frequentist or Bayesian), and the inference approach (i.e., parameter estimation or model selection). Another reason may be that previous studies have only attempted to make group-level inferences about the effect of emphasizing urgency, in which a single conclusion is drawn for all participants in the experiment (see Evans, 2019 for a discussion). However, previous research has shown that relying solely on group-level inferences can be deceptive, as these approaches ignore individual differences in the decision-making process, and may lead to a minority of participants with strong evidence for one conclusion overwhelming a majority of participants with weaker evidence for another conclusion (Evans, Hawkins, \& Brown, 2020). A third reason may be that previous studies using group-level model selection for inferences did not specify different models for decreases and increases in decision-making components, "scoring key alternative 1" that assumed speed instructions should only decrease both threshold and drift rate. 
and instead compared a null model to an effect model and decided the direction of the effect using group-level parameter estimates (e.g., Rae et al., 2014, and several teams in Dutilh et al., 2019). Importantly, this approach results in participants who show strong decreases and increases in a decision-making component supporting the same model - the effect model - meaning that evidence for an effect may be due to an aggregation of participants showing both decreases and increases. One final reason may be differences in what results are interpreted as important and/or meaningful. For example, the results of Rae et al. (2014) showed changes in both drift rate and non-decision time in most cases, though the key interpretation of the findings - reflected by the alternative scoring key in Dutilh et al. (2019) - only involve the change in drift rate.

The current study aims to provide a more conclusive answer to which components of the decision-making process are influenced by emphasizing urgent responding. Specifically, the current study uses a common statistical framework to re-analyse 6 data sets from previous studies that used a manipulation of speed and accuracy emphasis instructions (Dutilh et al., 2013, 2019; Evans et al., 2017; Lerche \& Voss, 2017; Rae et al., 2014; Wagenmakers, Ratcliff, Gomez, \& McKoon, 2008), allowing for an assessment of whether different studies seem to provide different conclusions. The current study performs the reanalysis using Bayes factors (Kass \& Raftery, 1995), a state-of-the-art method for model selection based inference in the field of mathematical psychology (Evans \& Brown, 2018; Annis, Evans, Miller, \& Palmeri, 2019; Evans \& Annis, 2019). To avoid evidence for decreases and increases in a parameter value being interpreted as evidence for the same model, the current study defined separate models for positive and negative effects, which can naturally be done within the Bayesian framework using prior distributions that are truncated to positive-only and negative-only values, respectively (Haaf \& Rouder, 2017). Furthermore, the current study provides model-based inference on the level of individual 
participants, in order to ensure that group-level inferences are not dominated by a minority of participants with a strong effect for one conclusion, with broader conclusion being made based on the general trends across participants (Evans, Hawkins, \& Brown, 2020). Finally, the current study applies two different EAMs - the diffusion model (Stone, 1960; Ratcliff, 1978) and the linear ballistic accumulator (LBA; Brown \& Heathcote, 2005, 2008) - in order to assess whether different EAMs lead to different conclusions about what components of the decision-making process are influenced by emphasizing urgency.

\section{Method}

The current study provides an assessment of which EAM parameters differ when urgency is emphasised through the use of speed and accuracy emphasis instructions. The current assessment uses 6 data sets from 6 different previous studies: Dutilh et al. (2013, 2019); Evans et al. (2017); Lerche and Voss (2017); Rae et al. (2014); Wagenmakers et al. (2008). Here, I provide a brief description of each of these data sets, followed by detailed descriptions of the analysis framework and the formal models used within the current study.

\section{Description of data sets}

For all data sets, the current study excluded trials that were faster than 0.15 seconds from the analyses. For the Lerche and Voss (2017) data set, which was specifically designed as a more complex task with longer response times, the current study excluded trials that were slower than 30 seconds, and for all other data sets the current study excluded trials that were slower than 5 seconds. In all cases, participant exclusions were the same as in the original studies.

Dutilh et al. (2013). The current study assesses Experiment 1 of Dutilh et al. (2013), where 32 participants completed a random dot motion task (Shadlen \& Newsome, 1996; 
Pilly \& Seitz, 2009), with different alternating blocks of trials having either speed or accuracy emphasis instructions. It should also be noted that participants were additionally given response deadlines in speed emphasis blocks, which were adapted to each participant according to their estimated non-decision time in practice blocks of trials. As Dutilh et al. (2013) were interested in the effects of ageing, these participants consisted of 15 younger adults and 17 older adults. However, as the current study does not focus on age-related differences, an explicit distinction is not made between young and old participants. Participants spent two hours on the task, meaning that the number of trials completed differed between conditions and participants: the range of the number of trials completed in the speed emphasis condition was 102-923, and the range in the accuracy emphasis was 22-440.

Dutilh et al. (2019). The data of Dutilh et al. (2019) consists of a large experiment where 20 participants completed factorial manipulations of urgency emphasis, stimulus difficulty, and response bias. However, the goal of Dutilh et al. (2019) was to assess the ability of different groups of researchers to make inferences about different blinded experimental manipulations, which resulted in the organizers of the study splitting the large experiment into 14 different two-condition "pseudo-experiments" that differed in one or more manipulation(s). The current study assesses the 3rd pseudo-experiment of Dutilh et al. (2019), where the two conditions only differed in urgency emphasis. Each participant completed 200 trials per condition of a random dot motion task, where urgency emphasis was manipulated between blocks with speed or accuracy emphasis instructions. It should also be noted that participants were additionally informed that their response was "too slow" if they took longer than 0.8 seconds in speed emphasis blocks, though there was not an explicit deadline on the responses.

Evans et al. (2017). The current study assesses the first experiment of Evans et al. (2017), where 49 participants completed a brightness discrimination task (Ratcliff \& 
Rouder, 1998), deciding whether a black-and-white stimulus square was dominated by either black or white pixels. As Evans et al. (2017) excluded two participants for poor performance, the current study also excluded these participants, leaving 47 participants for the analyses. Alternating blocks provided either speed or accuracy emphasis instructions for participants as the manipulation of urgency emphasis. Participants completed 208 trials per condition, which was split in 2 blocks of 104 trials each. Note that participants also completed a questionnaire assessing their need for closure (Webster \& Kruglanski, 1994), though this is not discussed any further as it is not relevant to the current study.

Lerche and Voss (2017). The current study assesses Experiment 1 of Lerche and Voss (2017), where 38 participants completed a figural task, deciding whether one set of rectangles had a greater total area than another set of rectangles. Participants performed blocks either under speed or accuracy emphasis instructions, with accuracy emphasis blocks also rewarding participants for correct responses, and speed emphasis blocks also rewarding participants for fast responses. Participants completed 82 trials per condition, with all trials for a condition being completed in a single block, and the order of the blocks being counterbalanced across participants. Note that one of the primary goals of Lerche and Voss (2017) was to assess the empirical validity of diffusion model assessments of more complex tasks that take a longer time to complete, and therefore, the stimuli in Lerche and Voss (2017) consisted of multiple elements that participants had to process and resulted in much longer response times than the other experiments assessed within the current study. It should also be noted that participants completed another block of trials with accuracy emphasis instructions and an easier set of trials, which acted as an assessment of the selective influence of difficulty manipulations, though this additional block of trials is not analysed within the current study. 
Rae et al. (2014). The current study assesses Experiment 1 of Rae et al. (2014), where 49 participants completed a brightness discrimination task. As Rae et al. (2014) excluded 14 participants for poor performance, the current study also excludes these participants, leaving 35 participants for the analyses. Participants completed either 1 hour or 2 hours of trials, with each hour consisting of 14 blocks of 104 trials. Alternating blocks provided either speed or accuracy emphasis instructions for participants as the manipulation of urgency emphasis, meaning that participants completed either 728 or 1456 trials per condition. It should also be noted that participants were additionally informed that their response was "too slow" if they took longer than 0.8 seconds in speed emphasis blocks, though there was not an explicit deadline on the responses. Note that participants also completed response signal trials where they were prompted for a response at a certain time interval, though as these trials could induce different decision strategies (e.g., collapsing thresholds; Evans, Hawkins, \& Brown, 2020; Evans \& Hawkins, 2019), they are not analyzed within the current study.

Wagenmakers et al. (2008). The current study assesses Experiment 1 of Wagenmakers et al. (2008) - which was also analyzed as Experiment 2 of Rae et al. (2014) - where 17 participants completed a lexical decision task. Alternating blocks provided either speed or accuracy emphasis instructions for participants as the manipulation of urgency emphasis. Participants completed 960 trials per condition, which was divided into 10 blocks of 96 trials each. It should also be noted that participants were additionally informed that their response was "too slow" if they took longer than 0.75 seconds in speed emphasis blocks, though there was not an explicit deadline on the responses. Note that participants also completed trials of differing difficulty, being high frequency words, low frequency words, very low frequency words, and non-words, which were modelled by allowing drift rate to vary over these difficulties. Therefore, within this data set, the influence of 
urgency emphasis on drift rate was assessed as whether there was an additional additive difference between speed and accuracy emphasis conditions in drift rate.

\section{Analysis framework}

The current study assesses whether emphasizing urgency influences drift rate, decision threshold, and/or non-decision time through a model selection approach. Model selection approaches involve representing the different discrete possibilities (i.e., the different potential conclusions) as different models, and comparing the adequacy of the different models in explaining the empirical data through some quantitative metric (see Evans, 2019 and Evans, 2020 for discussions). Model selection approaches differ from parameter estimation approaches, which instead involve estimating the parameters of the model and performing some type of inference on the estimated values. Although both approaches have their strengths and weaknesses (Kruschke, 2013; Kruschke \& Liddell, 2018; Hoekstra, Morey, Rouder, \& Wagenmakers, 2014; van Ravenzwaaij \& Wagenmakers, 2019), the current study uses a model selection approach as these approaches (1) provide a clear measure of evidence for whether or not an effect exists (Wagenmakers, 2007), and (2) are less vulnerable to inference issues in complex models with highly correlated parameters that have strong tradeoffs (Evans, Trueblood, \& Holmes, 2020; i.e., the "sloppy models" problem Gutenkunst et al., 2007; Holmes, 2015).

The current study uses Bayesian model selection (e.g., Bayes factors; Kass \& Raftery, 1995) for inference, which through recent developments can be estimated for complex cognitive models (Evans \& Brown, 2018; Annis et al., 2019; Evans \& Annis, 2019; Gronau, Heathcote, \& Matzke, 2019). The choice of Bayesian model selection was based on their property of statistical consistency (Gronau \& Wagenmakers, 2019), whereas other commonly used methods that assess out of sample predictive accuracy show biases towards detecting effects (Evans, 2019). Specifically, the marginal likelihoods (i.e., the probability 
of the data given a model) were estimated through bridge sampling (Gronau et al., 2017), which uses samples from the posterior distribution, samples from a proposal distribution that is similar to the posterior distribution, and a bridge function between them. Posterior distributions were estimated through differential evolution Markov chain Monte Carlo (DEMCMC; Ter Braak, 2006; Turner, Sederberg, Brown, \& Steyvers, 2013), with $3 k$ parallel chains that were each run for 11,000 iterations (where $k$ is the number of free parameters in the model), with the first 1,000 iterations discarded as burn-in.

Previous cognitive modelling studies (e.g., Rae et al., 2014, several teams in Dutilh et al., 2019) using model selection approaches have only assessed whether or not a manipulation (e.g., emphasizing urgency) has an effect on a parameter of interest (e.g., decision threshold). However, these assessments ignore the direction of the effect, meaning that evidence for a decrease in the parameter and evidence for an increase in the parameter both show evidence in favour of the same model: the "effect" model. Importantly, decreases and increases in a parameter value have very different theoretical interpretations, and therefore, should be represented by different discrete possibilities (Haaf \& Rouder, 2017). The current study addresses this issue by defining the direction of the effect in each model that contained an effect, meaning that models either reflected a decrease, an increase, or no change in a parameter when assessing how speed emphasis instructions differ from accuracy emphasis instructions. Therefore, when considering all combinations of decision threshold, drift rate, and non-decision time each either decreasing, increasing, or being unchanged, there were 27 different models of how emphasizing urgency might influence the decision-making process. Furthermore, the current study provided inferences at the level of individual participants, preventing the potential issues that can arise from ignoring individual differences (Evans, Hawkins, \& Brown, 2020). General conclusions were made based upon the general pattern of inferences across participants, factoring in both 
the preferred model and the strength of evidence in favour of it.

Inferences were made using probability weights for each model calculated from the marginal likelihoods (similar to AIC weights; Wagenmakers \& Farrell, 2004). Specifically, the probability weight for each of the 27 models was given by:

$$
W_{a, b, c}=\frac{M L_{a, b, c}}{\sum_{i=1}^{N} \sum_{j=1}^{N} \sum_{k=1}^{N} M L_{i, j, k}}
$$

where $W$ is the probability weight for a model, $M L$ is the marginal likelihood for a model, $a$ and $i$ index the potential decision threshold effects, $b$ and $j$ index the potential drift rate effects, $c$ and $k$ index the potential non-decision time effects, and $N$ is the number of different potential effect types (i.e., decreasing, increasing, and no change).

Although the comparison of 27 different candidate models for each participant in 6 different data sets provides a robust assessment of how emphasizing urgency influences decision-making, it also provides a great deal of information, which could be difficult to interpret and would require several tables and/or figures to display using presentation methods from previous studies (e.g., Rae et al., 2014; Evans, Hawkins, \& Brown, 2020). In an attempt to summarise the results as efficiently as possible, the current study displays the probability weights as cumulative bar plots, with different colours and different plots within a row referring to different models. Each row of plots in Figures 1 and 2 present a different study, whereas different plots within each row show the probability weights for models where threshold decreases (left), is unchanged (middle), or increases (right) when urgency is emphasized. Within each plot, the 9 combinations of models where drift rate and non-decision time decrease, are unchanged, or increase are represented by 9 different colours: purple (both decreasing), red (drift rate decreasing, non-decision time unchanged), cream (drift rate decreasing, non-decision time increasing), blue (drift rate unchanged, 
non-decision time decreasing), black (both unchanged), green (drift rate unchanged, nondecision time increasing), pink (drift rate increasing, non-decision time decreasing), orange (drift rate increasing, non-decision time unchanged), and yellow (both increasing). Each bar within a plot represents a different participant within the study, and the corresponding bar for the different plots within each row represent the same participant, meaning that all corresponding bars within a row of plots sum to a total probability of 1 . The ordering of participants was determined by the ratio of the average marginal likelihood for models with both drift rate and non-decision time decreasing and the average marginal likelihood for the models with both drift rate and non-decision time unchanged, in an attempt to make visual inspections of the figures easier. Importantly, this format allows for the comparison of the 27 different candidate models for each participant in the 6 different data sets to displayed in a single figure, with separate figures for comparisons using the diffusion model and the LBA.

Figure 3 displays an example of these plots using the diffusion model assessment of the Dutilh et al. (2013) data set (i.e., the top row of Figure 1), as well as a legend that shows the colours that correspond to the probability weight of each model. As a concrete example, consider the first bar in each of the three plots (i.e., the first participant): the largest probability weight for this participant is the purple bar in the second plot, meaning that the best explanation for how this participant's performance changes from accuracy emphasis instructions to speed emphasis instructions is a decrease in drift rate and nondecision time, and no change in decision threshold. Furthermore, the probability weight for this model is close to 1 , with only a small weight assigned to the purple bar in the first plot (i.e., all parameters decreasing) and essentially no weight assigned to the other models, meaning that the best model is strongly preferred over all other models. As a second concrete example, consider the second bar in each of the three plots (i.e., the second 
participant): the largest probability weight for this participant is the red segment of the bar in the first plot, meaning that the best explanation for how this participant's performance changes from accuracy emphasis instructions to speed emphasis instructions is a decrease in drift rate and decision threshold, and no change in non-decision time. However, the probability weight for this model is only around 0.5 , and is only slightly larger than the probability weight for the purple segment of the same bar, meaning that the best model is only slightly preferred over the model with all parameters decreasing. Nevertheless, the probability weights of these two models almost sum to 1 , meaning that these two models are strongly preferred over all other models, and that there is strong evidence that this participant's performance is best explained by decreases in both drift rate and decision threshold.

\section{Formal models}

The current study assesses whether emphasizing urgency influences drift rate, decision threshold, and/or non-decision time using two different EAMs: the diffusion model (Ratcliff, 1978) and the LBA (Brown \& Heathcote, 2008). Importantly, this allows for an assessment of whether different cognitive models lead to different conclusions about the effect of emphasizing urgency. Although previous research has suggested that these two models provide the same conclusions about psychological processes (Donkin, Brown, Heathcote, \& Wagenmakers, 2011), meaning that these models should not differ in their inferences, the many-labs project of Dutilh et al. (2019) showed a general pattern of differences between the conclusions of researchers who applied the LBA and researchers who applied the diffusion model, though these differences may have been the result of other differences in methodology (Evans, 2019). The diffusion and the LBA fall within the same general class of models (i.e., EAMs), and both propose the same general psychological process where decisions are the result of evidence accumulating to a decision threshold. 
However, the precise dynamics of the two models differ in several respects. Here, I provide a brief description of the differences between these models, as well as the exact model definitions used within the current study. Also note that the current study used a contamination process for both models (Ratcliff \& Tuerlinckx, 2002), where the probability density function for each model was assumed to be a mixture of the true probability density function $\left(1-p_{c}\right)$ and a contaminant distribution $\left(p_{c}\right)$. The contaminant distribution was assumed to be a uniform distribution over the range in response times allowed within the data sets (30 seconds for Lerche and Voss (2017), 5 seconds for all other data sets) for both correct and errors responses, and the probability of contamination $p_{c}$ was estimated as a free parameter of each model.

Diffusion model. The diffusion model proposes that evidence accumulation is a dependent process: specifically, that evidence for one alternative is evidence against the other alternative, and vice versa. This assumption means that the evidence accumulation process can be expressed using only 4 parameters: the difference in accumulation rate between the two alternatives $(v)$, the difference between the two thresholds $(a)$, the starting point of the accumulation $(z)$, and the non-decision time $\left(t_{e r}\right)$. The diffusion model also includes random moment-to-moment noise in the evidence accumulation process, represented by random draws from a normal distribution with mean 0 and standard deviation $s$, though $s$ is fixed to 1 to solve a scaling issue within the model. The current study involves assessments on the parameters that relate to the decision threshold $(a)$, drift rate $(v)$, and the non-decision time $\left(t_{e r}\right)$. Although some previous studies have also incorporated parameters that provide between-trial variability in drift rate $\left(s_{v}\right.$; Ratcliff, 1978), starting point $\left(s_{z}\right.$; Ratcliff \& Rouder, 1998), and non-decision time ( $s_{t e r}$; Ratcliff \& Tuerlinckx, 2002), the currently study only uses the "simple" (i.e., 4 parameter) diffusion model due to the poor measurement properties associated with the "full" (i.e., including all between-trial variabil- 
ity parameters) diffusion model (van Ravenzwaaij \& Oberauer, 2009; Lerche \& Voss, 2016; Lerche, Voss, \& Nagler, 2017; Boehm et al., 2018; Evans, Tillman, \& Wagenmakers, 2020). It should be noted that previous studies have shown the robust measurement properties of between-trial variability in non-decision time (i.e., $s_{t e r}$ ), and that the poor measurement properties associated with the "full" diffusion model appear to be due to the between-trial variability in drift rate and starting point (i.e., $s_{v}$ and $s_{z}$; van Ravenzwaaij \& Oberauer, 2009; Lerche \& Voss, 2016; Lerche et al., 2017). However, the main benefit of including between-trial variability in non-decision time is to reduce the susceptibility of the model to fast contaminants (Lerche \& Voss, 2016; Lerche et al., 2017), which the current study instead captures through a contaminant process (Ratcliff \& Tuerlinckx, 2002).

The probability density function of the model was calculated using code extracted from the fastdm software (Voss \& Voss, 2007). As the only parameters of theoretical interest within the current study are $v, a$, and $t_{e r}$, the parameters $z$ and $p_{c}$ (i.e., the contaminant parameter) were fixed to take the same values over emphasis conditions for parsimony. The parameters allowed to vary over emphasis (i.e., $v, a$, and $t_{e r}$ ) were parameterized as a mean and difference across conditions. This parameterization is important when performing Bayesian model selection, as the priors on the "intercept" (i.e., the mean) parameters have little influence on marginal likelihood - provided that they are not too narrow - whereas the priors on the "effect" (i.e., the difference) parameters can have a large impact on the marginal likelihood (Rouder, Morey, Speckman, \& Province, 2012). Note that a mean parameter across conditions was estimated for every parameter in every model, and that a difference parameter was only included for the parameters in each model that varied over emphasis conditions.

The priors for the mean parameter values over conditions were: 


$$
\begin{aligned}
v & \sim N(3,3) \\
t_{e r} & \sim T N(0.3,0.3,0, \infty) \\
a & \sim T N(2,2,0, \infty) \\
\frac{z}{a} & \sim T N(0.5,0.3,0,1) \\
p_{c} & \sim U(0,1)
\end{aligned}
$$

where $N$ is the normal distribution with parameters mean, standard deviation, $T N$ is the truncated normal distribution with parameters mean, standard deviation, lower truncation, upper truncation, and $U$ is the uniform distribution with parameters lower truncation, upper truncation. These priors were used for all data sets except for Lerche and Voss (2017), due to the longer response times creating more uncertainty in the potential parameter values, where the standard deviations of the priors were doubled.

The priors for the difference parameter values in models where the parameter was decreasing or increasing were:

$$
\begin{aligned}
v & \sim T N(0,3) \\
t_{e r} & \sim T N(0,3) \\
a & \sim T N(0,3)
\end{aligned}
$$

where the truncated normal distribution for each parameter was truncated between $-\infty$ and 0 for models where the parameter was decreasing, and truncated between 0 and $\infty$ for models where the parameter was increasing. These priors were used for all data sets. Note 
that the 0 centred priors for the difference parameters reflects an "objective" or "default" choice in prior (e.g., Rouder et al., 2012).

Linear ballistic accumulator. The LBA proposes that evidence accumulation is an independent process: specifically, that evidence for one alternative does not impact the evidence for the other alternative, and vice versa. This assumption means that the LBA has separate parameters for each accumulator, and that the evidence accumulation process for each accumulator can be expressed using only 3 parameters: the accumulation rate $(v)$, the decision threshold $(b)$, and the non-decision time $\left(t_{0}\right.$; similar to the $t_{e r}$ parameter in the diffusion model). Unlike the diffusion model, the LBA assumes that there is no momentto-moment noise in the evidence accumulation process. Instead, there is only between-trial variability in accumulation, represented by random draws from a normal distribution with mean $v$ and standard deviation $s$, though $s$ is fixed to 1 to solve a scaling issue within the model. Furthermore, the LBA assumes that there is between-trial variability in the starting amount of evidence, represented by random draws from a uniform distribution between 0 and $A$. Unlike the diffusion model, previous research has suggested that these sources of between-trial variability do not compromise the measurement properties of the model (Brown \& Heathcote, 2008; Donkin et al., 2011). As is standard practice, the $A$ and t0 parameters were fixed to take the same values over accumulators. The drift rate parameter $(v)$ was allowed to vary between accumulators, which was parameterized as the midpoint of the two drift rate $\left(v_{m i d}\right.$; the average overall accumulation rate) and the separation between the two drift rates $\left(v_{\text {sep }}\right.$; similar to the $v$ parameter in the diffusion model). The threshold parameter was also allowed to vary between accumulators, which was parameterized as the midpoint of the two thresholds ( $b_{\text {mid }}$; the average overall threshold, similar to the $a$ parameter in the diffusion model), and the separation between the two thresholds $\left(b_{\text {sep }}\right.$; the bias for one response option over the other, similar to the $z$ parameter in the diffusion 
model).

As the only parameters of theoretical interest within the current study are $v_{s e p}$, $b_{m i d}$, and $t 0$, the parameters $A$ and $b_{s e p}$ were fixed to take the same values over emphasis conditions for parsimony. However, following the finding of Rae et al. (2014) that $v_{\text {mid }}$ increased under urgency emphasis whereas $v_{\text {sep }}$ decreased, we allowed $v_{\text {mid }}$ to freely (i.e., no decreasing/increasing restrictions) vary over emphasis conditions in all models to ensure that it did not interfere with the inferences on $v_{\text {sep }}$. As with the diffusion model, the parameters of theoretical interest (i.e., $v_{s e p}, b_{m i d}$, and $t 0$ ) were parameterized as a mean and difference across conditions.

The priors for the mean parameter values over conditions were:

$$
\begin{aligned}
v_{\text {sep }} & \sim T N(1.5,1.5,0, \infty) \\
t_{0} & \sim T N(0.3,0.3,0, \infty) \\
b_{\text {mid }} & \sim T N(2,2,0, \infty) \\
v_{\text {mid }} & \sim T N(3,3,0, \infty) \\
\frac{b_{\text {sep }}}{b_{\text {mid }}} & \sim T N(0.5,0.3,0,1) \\
A & \sim T N(2,2,0, \infty) \\
p_{c} & \sim U(0,1)
\end{aligned}
$$

These priors were used for all data sets except for Lerche and Voss (2017), due to the longer response times creating more uncertainty in the potential parameter values, where the standard deviations of the priors was doubled. The priors for the difference parameter values in the models where the parameter was decreasing or increasing were: 


$$
\begin{aligned}
v_{\text {sep }} & \sim T N(0,3) \\
t_{0} & \sim T N(0,3) \\
b_{\text {mid }} & \sim T N(0,3)
\end{aligned}
$$

These priors were used for all data sets.

Also note that Bayes factors can be quite sensitive to the choice of prior, particularly regarding the choice of prior on the difference parameters (Rouder et al., 2012). In order to assess the robustness of the findings of the current study to the choice of priors, I also performed both the diffusion model and LBA analyses using more tightly informed priors on the difference parameters:

Diffusion:

$$
\begin{aligned}
v & \sim T N(0,0.1) \\
t_{e r} & \sim T N(0,0.1) \\
a & \sim T N(0,0.1)
\end{aligned}
$$

\section{LBA:}

$$
\begin{aligned}
v_{\text {sep }} & \sim T N(0,0.1) \\
t_{0} & \sim T N(0,0.1) \\
b_{\text {mid }} & \sim T N(0,0.1)
\end{aligned}
$$

which can be seen in Figures 4 and 5. As expected, there is an overall trend for the detection of more effects than when using the less informed priors (Figures 1 and 2), as is often the 
case with increasingly informed priors (Evans, 2019). However, these additional effects appear to be a fairly equal split between increases and decreases in the parameters in most cases, suggesting that the more informed priors do not change the overall conclusions of the current study, and instead only push participants who show small increases or decreases in a parameter towards the respective model. Therefore, the general pattern of results does not appear to change based on the use of more informed priors, and they will not be discussed further within the current study.

\section{Results}

\section{Diffusion model}

Figure 1 displays the diffusion model analyses. Here, I discuss the results for each study separately, and provide a brief summary of the results at the end of the subsection.

For Dutilh et al. (2013), most bars are within the leftmost plot, suggesting a general decrease in threshold. In this data set the dominant segments of most bars are purple, red, or light yellow in colour, suggesting a general decrease in drift rate. The prominence of red segments suggests that many participants show evidence for no change in non-decision time, though it should be noted that some participants have large segments that are purple or blue (i.e., in favour of a non-decision time decrease), showing that there may be some individual differences. Therefore, the diffusion model assessment of Dutilh et al. (2013) appears to suggest that threshold and drift rate decrease under urgency emphasis, though non-decision time does not.

For Dutilh et al. (2019), most bars are within the leftmost plot, suggesting a general decrease in threshold. In this data set the dominant segment of most bars are purple, blue, or pink in colour (particularly for the few bars that are in the central plot), suggesting a general decrease in non-decision time (particularly in cases where threshold does not). The 
prominence of blue segments suggests that many participants show evidence for no change in drift rate, though it should be noted that some participants do have large segments that are purple or red (i.e., in favour of a drift rate decrease), showing that there may be some individual differences. Therefore, the diffusion model assessment of Dutilh et al. (2019) appears to suggest that threshold and non-decision decrease under urgency emphasis, though drift rate does not.

For Evans et al. (2017), almost every bar is within the leftmost plot, showing clear evidence for a general decrease in threshold. In this data set the dominant segment of many bars are purple, blue, or pink in colour, suggesting a general decrease in non-decision time, though the dominant segment of most other bars are black or orange, suggesting that many participants also show no decrease in non-decision time. Interestingly, there are very few segments that are purple, red, or light yellow, suggesting little evidence for a decrease in drift rate, and several segments that are pink or orange, suggesting some evidence for an increase in drift rate. Therefore, the diffusion model assessment of Evans et al. (2017) appears to suggest that threshold and non-decision decrease under urgency emphasis, though drift rate does not.

For Lerche and Voss (2017), most bars are within the leftmost plot, showing clear evidence for a general decrease in threshold. In this data set the dominant segment of many bars are purple, blue, or pink in colour, suggesting a general decrease in non-decision time. Interestingly, there are very few segments that are purple, red, or light yellow, suggesting little evidence for a decrease in drift rate. Therefore, the diffusion model assessment of Lerche and Voss (2017) appears to suggest that threshold and non-decision decrease under urgency emphasis, though drift rate does not.

For Rae et al. (2014), most bars are within the leftmost plot, suggesting a general decrease in threshold. In this data set the dominant segment of many bars are purple, 
blue or pink in colour (particularly for the bars that are in the central plot), suggesting a general decrease in non-decision time (particularly in cases where threshold does not). The prominence of purple segments suggests that many participants show evidence for a decrease in drift rate, though it should be noted that some participants have large segments that are blue (i.e., in favour of no change in drift rate), showing that there may be some individual differences. Therefore, the diffusion model assessment of Rae et al. (2014) appears to suggest that threshold, non-decision, and drift rate decrease under urgency emphasis.

For Wagenmakers et al. (2008), essentially all probability weights are within the leftmost plot, showing conclusive evidence for a general decrease in threshold. In this data set the dominant segment of many bars are purple, blue, or pink in colour, suggesting a general decrease in non-decision time, though the dominant segment of most other bars are black or orange, suggesting that many participants also show no decrease in non-decision time. Interestingly, although a few segments are purple, which would indicate a decrease in drift rate, most segments are blue or black, indicating no change in drift rate, and some segments are even being pink, which would indicate an increase in drift rate. Therefore, the diffusion model assessment of Wagenmakers et al. (2008) appears to suggest that threshold and non-decision time decrease under urgency emphasis, though drift rate does not.

Overall, the diffusion model analyses seem to indicate differences in conclusions between studies, and individual differences between participants in most studies. However, some general patterns did emerge. All studies show a general trend for a threshold decrease under urgency emphasis - as intuitively expected - though this was slightly less apparent in Rae et al. (2014). Five studies showed a general trend for non-decision time decreasing under urgency emphasis (Dutilh et al., 2019; Evans et al., 2017; Lerche \& Voss, 2017; Rae et al., 2014; Wagenmakers et al., 2008), though Dutilh et al. (2013) - for most participants - showed no change in non-decision time under urgency emphasis. Two studies showed a 
general trend for drift rate decreasing under speed emphasis (Dutilh et al., 2013; Rae et al., 2014), though four studies show a general trend for drift rate being unchanged (Dutilh et al., 2019; Lerche \& Voss, 2017; Wagenmakers et al., 2008) or even increasing (Evans et al., 2017). Therefore, the diffusion model analyses appear to indicate that threshold, and to a lesser extent non-decision time, decrease under urgency emphasis, though drift rate seems to remain unchanged in most cases.

\section{Linear ballistic accumulator}

Figure 2 displays the LBA analyses. Here, I discuss the results for each study separately, and provide a brief summary of the results at the end of the subsection.

For Dutilh et al. (2013), approximately half of the bars are within the leftmost plot, suggesting a general decrease in threshold, though that there also appears to be some individual differences. In this data set the dominant segments of most bars are purple, red, or light yellow in colour, suggesting a general decrease in drift rate. The prominence of red segments suggests that many participants show evidence for no change in non-decision time, with few participants having segments that are purple, blue, or pink. Therefore, the LBA assessment of Dutilh et al. (2013) appears to suggest that threshold and drift rate decrease under urgency emphasis, though non-decision time does not.

For Dutilh et al. (2019), just under half of the bars are within the leftmost plot, suggesting some evidence for a general decrease in threshold, though that most participants show no change in threshold. In this data set the dominant segments of most bars are purple, red, or light yellow in colour, suggesting a general decrease in drift rate, though it should be noted that some participants have large segments that are black (i.e., in favour of no change in drift rate), showing that there may be some individual differences. The prominence of red and black segments suggests that many participants show evidence for no change in non-decision time, with few participants having segments that are purple, 
blue, or pink. Therefore, the LBA assessment of Dutilh et al. (2019) appears to suggest that drift rate decreases under urgency emphasis, non-decision time does not, and that there is mixed evidence between a decrease and no change for threshold.

For Evans et al. (2017), most bars are within the central plot, suggesting a general lack of decrease in threshold, though it should be noted that some participants have large segments of their bars within the leftmost panel (i.e., in favour of a decrease in threshold), showing that there may be some individual differences. In this data set the dominant segments of most bars are black in colour, suggesting that most participants show evidence for no change in drift rate or non-decision time. However, it should be noted that some participants have large segments that are purple, red, or blue (i.e., in favour of a drift rate and/or non-decision time decrease), showing that there may be some individual differences. Therefore, the LBA assessment of Evans et al. (2017) appears to suggest that threshold, drift rate, and non-decision time do not change under urgency emphasis.

For Lerche and Voss (2017), most bars are within the leftmost plot, showing clear evidence for a general decrease in threshold. In this data set the dominant segments of most bars are black in colour, suggesting that most participants show evidence for no change in drift rate or non-decision time. However, it should be noted that some participants have large segments that are purple or blue (i.e., in favour of a drift rate and non-decision time decrease, or only a non-decision time decrease), showing that there may be some individual differences, particularly in non-decision time. Therefore, the LBA assessment of Lerche and Voss (2017) appears to suggest that threshold decreases under urgency emphasis, though drift rate and non-decision time do not.

For Rae et al. (2014), just under half of the bars are within the leftmost plot, suggesting some evidence for a general decrease in threshold, though that most participants show no change in threshold. In this data set the dominant segments of most bars are 
red or black in colour, suggesting that most participants show evidence for no change in non-decision time. However, it should be noted that some participants have large segments that are purple or blue (i.e., in favour of a non-decision time decrease), showing that there may be some individual differences. The many red segments suggest some evidence for a general decrease in drift rate, though more segments appear to be black, suggesting that most participants show no change in drift rate. Therefore, the LBA assessment of Rae et al. (2014) appears to suggest that non-decision time does not change under urgency emphasis, and that there is mixed evidence between a decrease and no change for threshold and drift rate.

For Wagenmakers et al. (2008), just over half of the bars are within the leftmost plot, suggesting a general decrease in threshold, though that there appears to be some individual differences. In this data set the dominant segments of most bars are purple, red, or light yellow in colour, suggesting a general decrease in drift rate. The prominence of red segments suggests that many participants show evidence for no change in nondecision time, though it should be noted that some participants have large segments that are purple (i.e., in favour of a decrease in non-decision time), showing that there may be some individual differences. Therefore, the LBA assessment of Wagenmakers et al. (2008) appears to suggest that threshold and drift rate decrease under urgency emphasis, though non-decision time does not.

Overall, the LBA analyses seems to indicate major differences in conclusions between studies, and individual differences between participants in most studies. However, some general patterns did emerge, though they appear to be much weaker than in the diffusion model analyses. Half of the studies show a general trend for a threshold decrease under urgency emphasis (Dutilh et al., 2013; Lerche \& Voss, 2017; Wagenmakers et al., 2008), though two of the studies showed mixed evidence between a decrease and no change (Dutilh 
et al., 2019; Rae et al., 2014), and one study showed evidence for no change (Evans et al., 2017). Half of the studies also show a general trend for drift rate decreasing under urgency emphasis (Dutilh et al., 2013, 2019; Wagenmakers et al., 2008), though one of the studies showed mixed evidence between a decrease and no change (Rae et al., 2014), and two studies showed evidence for no change (Evans et al., 2017; Lerche \& Voss, 2017). All studies show a general trend for no change in non-decision time under urgency emphasis, which is in striking contrast to the findings of the diffusion model analyses. Therefore, the LBA analyses appears to partially indicate that threshold and drift rate decrease under urgency emphasis - though these results were quite mixed - and strongly indicate that non-decision time remains unchanged under urgency emphasis.

\section{Discussion}

The current study aimed to provide a more conclusive answer on what components of the decision-making process are influenced by emphasizing urgent responding. The current study re-analysed 6 data sets with a manipulation of speed and accuracy emphasis instructions (Dutilh et al., 2013, 2019; Evans et al., 2017; Lerche \& Voss, 2017; Rae et al., 2014; Wagenmakers et al., 2008), with all analyses using the same state-of-the-art framework for model selection based inference in cognitive modelling. The overall pattern of results showed that participants generally had lower thresholds under speed emphasis than accuracy emphasis, indicating that people are less cautious when urgency is emphasized. This finding is in line with those of previous studies, and suggests that the emphasizing urgency does result in a decrease of decision thresholds, matching part of the intuitive expectations that researchers have had about the manipulation.

However, the overall pattern of results was less clear for drift rate and non-decision time. While there was evidence for a decrease in each parameter in some instances, these 
instances were less common than those for a decrease in threshold, suggesting that even if drift rate and non-decision time are lower under speed emphasis than accuracy emphasis in some participants and/or situations, these cases are much less common than the intuitively expected decrease in threshold. Furthermore, there were few instances where there was evidence for both non-decision time and drift rate decreasing, meaning that in cases where one parameter appeared to decrease, the other appeared to be unchanged. Importantly, these results indicate that the diverging conclusions of previous studies should not be interpreted as evidence for a decrease in threshold, drift rate, and non-decision time, as changes in drift rate and non-decision time were generally not found simultaneously.

Although the unclear pattern of results for drift rate and non-decision time were partially the result of differences between participants and between studies, the discrepancy in findings can mostly be explained through the differences in conclusions between the diffusion model and the LBA. Specifically, the diffusion model analyses showed that participants generally had lower thresholds under speed emphasis than accuracy emphasis in every study, and that participants generally had lower non-decision times under speed emphasis than accuracy emphasis in most studies. However, the diffusion model analyses also showed that participants generally had drift rates that did not change from accuracy to speed emphasis, with only a couple of studies showing participants to generally have lower drift rates under speed emphasis than accuracy emphasis. In contrast, the LBA analyses showed that participants generally had lower thresholds under speed emphasis than accuracy emphasis in half of the studies - with mixed evidence in most others that participants generally had lower drift rates under speed emphasis than accuracy emphasis in half of the studies - with mixed evidence in one other - and that participants generally had non-decision times that did not change from accuracy to speed emphasis in every study. These conclusions are in striking contrast with one another, with the diffu- 
sion model suggesting that emphasizing urgency decreases both threshold and non-decision time, and the LBA suggesting that emphasizing urgency decreases both threshold and drift rate. Importantly, these results indicate that conclusions about what components of the decision-making process are influenced by emphasizing urgency are heavily influenced by the choice of model, with the diffusion model suggesting that people become less cautious and faster at motor responding, and the LBA suggesting that people become less cautious and poorer at the task.

One interesting aspect of the LBA analyses was that for every study, many participants - and in some studies, most participants - showed strong evidence for no change in threshold under urgency emphasis. Furthermore, in half of the studies more participants showed evidence in favour of a decrease in drift rate than in favour of a decrease in threshold. This is in contrast to the diffusion model analyses, where in all studies most participants showed strong evidence for a decrease in threshold under urgency emphasis. The findings of the LBA analyses appear odd, given that the intuitions of most researchers, and the conclusions of all previous studies, are that at the very least threshold decreases under urgency emphasis, and in some cases other parameters change as well. Furthermore, the findings of the diffusion model analyses are in line with these general intuitive expectations about threshold, suggesting that the odd findings of the LBA analyses are not consistent across models. Therefore, based on the somewhat odd conclusions of the LBA, researchers may wish to place more weight on the conclusions of the diffusion model, which indicated that emphasising urgency results in a decrease in both threshold and non-decision time.

The discrepancy in conclusions between the diffusion model and the LBA observed within the current study contrast with previous studies that have suggested that these models come to the same conclusions, such as Donkin et al. (2011). Donkin et al. (2011) performed a cross-fit simulation study, where the simulated data sets were generated from 
one model and the parameters were estimated from the simulated data using the other model, finding a close correspondence between the generating values of each model and the estimated parameters of the other model in most cases. However, the simulation set-up of Donkin et al. (2011) differed from the type of experimental set-up that researchers often wish to analyse in practice (e.g., Dutilh et al., 2019). Specifically, Donkin et al.'s simulation set-up included a single within-subjects condition that only differed in the generated drift rate, and the models fit to these data were constrained to only allow drift rate to vary between these conditions, which provides additional constraint on the models and may lead them to similar conclusions. This is in contrast to most empirical studies where researchers are interested in determining which parameters vary over within-subjects conditions such as in the current study - which does not provide the additional constraint used in the simulation study, and therefore, might more easily showcase differences between the models in their conclusions.

However, this cannot be the primary reason for why the conclusions of the current study differ from Donkin et al. (2011), as Donkin et al. (2011) also performed assessments of previous empirical studies with both models, allowing all parameters to freely vary over within-subjects condition, and finding that the models largely came to the same conclusions about which parameters varied over conditions. However, the definition of the LBA in Donkin et al. (2011) differed from the definition of the LBA that researchers often use in practice (e.g., Rae et al., 2014; Dutilh et al., 2019). Specifically, Donkin et al.'s LBA fitting constrained the drift rates of the correct and error evidence accumulators to sum to 1 (i.e., constraining the average accumulation rate to be 0.5 ), which removes the need to estimate the average accumulation rate, making the LBA more similar to the diffusion model. This is in contrast to most empirical studies where researchers estimate both the average accumulation rate and the difference in accumulation rate (e.g., Rae et 
al., 2014; Dutilh et al., 2019) - or, equivalently, estimate separate drift rates for each accumulator - which provides less constraint and provides the LBA with an additional decision-making component that is not present in the diffusion model. Therefore, the key difference in whether or not the conclusions of the diffusion model and the LBA agree may be dependent on the specific definition of the LBA, and whether or not the average accumulation rate is freely estimated. Future research should provide a more detailed assessment of the different potential definitions of the LBA, and whether these different definitions change the conclusions of the model.

Although the diffusion model and the LBA came to several different conclusions within the current study, there is one important conclusion that they agree on: that there is a violation of the selective influence assumption for emphasizing urgency through speed and accuracy instructions. Specifically, the diffusion model suggested that both threshold and non-decision time were lower under speed emphasis than accuracy emphasis, and the LBA suggested that both threshold and drift rate were lower under speed emphasis than accuracy emphasis. Although each model suggests that a different parameter varies in addition to threshold, both models suggest that an additional parameter varies, showing clear evidence that speed and accuracy instructions do not selectively influence threshold within these models. Therefore, researchers should no longer assume that this manipulation only influences threshold, such as in Dutilh et al. (2019).

Importantly, the clear violation of the selective influence assumption observed within the current study raises the question of why this violation occurs. Importantly, this violation could indicate a problem with the models, where they are detecting spurious effects, or a problem with the assumption underlying the manipulation, where the manipulation is not a valid selective manipulation of what people believe it should selectively manipulate. Experts in the field mostly seem to believe that this reflects a failing of the assumptions of 
the manipulation, and that emphasizing urgency is unable to selectively influence threshold (Voss et al., 2004; Rae et al., 2014; Lerche \& Voss, 2017), though some have argued that the manipulation is a valid one, and that the models are merely detecting spurious effects as they are only crude approximations of the true underlying decision-making process (Dutilh et al., 2019). The current study does not provide any evidence to adjudicate between these two explanations for why the selective influence assumption fails, though I attempt to provide two potential avenues for future research that may help to resolve this question.

The first potential avenue of research is to search for different manipulations of response caution, which are also expected to selectively influence this component of the decision-making process. As discussed in the introduction, it seems plausible that emphasizing urgent responding could have unintended consequences on other components of the decision-making process, meaning that this manipulation may be poorer at selectively influencing threshold than other possible manipulations. Importantly, if other manipulations were found that were able to selectively influence threshold, then it would indicate that the reason for the violation of the selective influence assumption when emphasizing urgency was a failure of researcher intuitions about the manipulation, rather than a failing of the models, as it would provide evidence that threshold can be selectively influenced with a better manipulation. However, finding a selective manipulation of threshold may prove more difficult than for drift rate. Specifically, as threshold is thought to be largely under the control of the participants, any manipulations of threshold must somehow convince participants to change their threshold while also leaving all aspects of the process unchanged. This is in contrast to drift rate, which is thought to be largely outside of the control of participants, and therefore, selective manipulations can easily be created by changing the difficulty of the task. Therefore, attempting to find a different, selective 
manipulation of threshold may be a difficult task, though finding a selective manipulation would conclusively display that threshold can be selectively influenced with a better manipulation.

The second potential avenue of research is to design new process models that are able to account for the effects of emphasizing urgency with only changes in a parameter relating to response caution. Although both the diffusion model and the LBA suggest that emphasizing urgency changes response caution as well as another component of the decision-making process, this may be a result of model misspecification. As all cognitive models are likely crude approximations to the underlying cognitive processes (Box, 1976), there is likely a great deal of misspecification in both the diffusion model and the LBA, which could result in misleading inferences in some circumstances. Although there is no specific reason to believe that model misspecification is the reason for urgency emphasis manipulations failing to selectively influence response caution - at least, no more than any other manipulation - developing a model that is able to account for the effects of emphasizing urgency with only changes in a parameter relating to response caution would conclusively display that emphasizing urgency can selectively influence response caution with a better model. 


\section{References}

Annis, J., Evans, N. J., Miller, B. J., \& Palmeri, T. J. (2019). Thermodynamic integration and steppingstone sampling methods for estimating bayes factors: A tutorial. Journal of mathematical psychology, 89, 67-86.

Boehm, U., Annis, J., Frank, M. J., Hawkins, G. E., Heathcote, A., Kellen, D., ... Wagenmakers, E.-J. (2018). Estimating across-trial variability parameters of the diffusion decision model: Expert advice and recommendations. Journal of Mathematical Psychology, 87, 46-75.

Bogacz, R., Brown, E., Moehlis, J., Holmes, P., \& Cohen, J. D. (2006). The physics of optimal decision making: A formal analysis of models of performance in two-alternative forced-choice tasks. Psychological Review, 113(4), 700.

Box, G. E. (1976). Science and statistics. Journal of the American Statistical Association, 71 (356), 791-799.

Brown, S. D., \& Heathcote, A. (2005). A ballistic model of choice response time. Psychological review, 112(1), 117.

Brown, S. D., \& Heathcote, A. (2008). The simplest complete model of choice response time: Linear ballistic accumulation. Cognitive psychology, 57(3), 153-178.

Crüwell, S., Stefan, A. M., \& Evans, N. J. (2019). Robust standards in cognitive science. Computational Brain \& Behavior, 2(3-4), 255-265.

Donkin, C., Averell, L., Brown, S., \& Heathcote, A. (2009). Getting more from accuracy and response time data: Methods for fitting the linear ballistic accumulator. Behavior research methods, 41(4), 1095-1110.

Donkin, C., Brown, S., Heathcote, A., \& Wagenmakers, E.-J. (2011). Diffusion versus linear ballistic accumulation: different models but the same conclusions about psychological processes? Psychonomic bulletin \& review, 18(1), 61-69.

Dutilh, G., Annis, J., Brown, S. D., Cassey, P., Evans, N. J., Grasman, R. P., ... Donkin, C. (2019). The quality of response time data inference: A blinded, collaborative assessment of the validity of cognitive models. Psychonomic Bulletin \& Review, 26(4), 1051-1069.

Dutilh, G., Forstmann, B. U., Vandekerckhove, J., \& Wagenmakers, E.-J. (2013). A diffusion model 
account of age differences in posterror slowing. Psychology and Aging, 28(1), 64 .

Evans, N. J. (2019). Assessing the practical differences between model selection methods in inferences about choice response time tasks. Psychonomic Bulletin \& Review, 26(4), 1070-1098.

Evans, N. J. (2020). What factors are most important in finding the best model of a psychological process? comment on navarro (2018). Meta-Psychology.

Evans, N. J., \& Annis, J. (2019). Thermodynamic integration via differential evolution: A method for estimating marginal likelihoods. Behavior research methods, 51(2), 930-947.

Evans, N. J., Bennett, A. J., \& Brown, S. D. (2019). Optimal or not; depends on the task. Psychonomic bulletin \& review, 26(3), 1027-1034.

Evans, N. J., \& Brown, S. D. (2018). Bayes factors for the linear ballistic accumulator model of decision-making. Behavior research methods, 50(2), 589-603.

Evans, N. J., \& Hawkins, G. E. (2019). When humans behave like monkeys: Feedback delays and extensive practice increase the efficiency of speeded decisions. Cognition, 184, 11-18.

Evans, N. J., Hawkins, G. E., \& Brown, S. D. (2020). The role of passing time in decision-making. Journal of Experimental Psychology: Learning, Memory, and Cognition.

Evans, N. J., Rae, B., Bushmakin, M., Rubin, M., \& Brown, S. D. (2017). Need for closure is associated with urgency in perceptual decision-making. Memory $\&$ cognition, 45(7), 11931205.

Evans, N. J., \& Servant, M. (2020). A comparison of conflict diffusion models in the flanker task through pseudolikelihood bayes factors. Psychological Review, 127(1), 114.

Evans, N. J., Steyvers, M., \& Brown, S. D. (2018). Modeling the covariance structure of complex datasets using cognitive models: An application to individual differences and the heritability of cognitive ability. Cognitive science, 42(6), 1925-1944.

Evans, N. J., Tillman, G., \& Wagenmakers, E.-J. (2020). Systematic and random sources of variability in perceptual decision-making: Comment on ratcliff, voskuilen, and mckoon (2018). Psychological Review.

Evans, N. J., Trueblood, J. S., \& Holmes, W. R. (2020). A parameter recovery assessment of time-variant models of decision-making. Behavior research methods, 52(1), 193-206.

Evans, N. J., \& Wagenmakers, E.-J. (2020). Evidence accumulation models: Current limitations 
and future directions. The Quantitative Methods for Psychology.

Gronau, Q. F., Heathcote, A., \& Matzke, D. (2019). Computing bayes factors for evidenceaccumulation models using warp-iii bridge sampling. Behavior research methods, 1-20.

Gronau, Q. F., Sarafoglou, A., Matzke, D., Ly, A., Boehm, U., Marsman, M., ... Steingroever, H. (2017). A tutorial on bridge sampling. Journal of mathematical psychology, 81, 80-97.

Gronau, Q. F., \& Wagenmakers, E.-J. (2019). Limitations of bayesian leave-one-out cross-validation for model selection. Computational brain \& behavior, 2(1), 1-11.

Gutenkunst, R. N., Waterfall, J. J., Casey, F. P., Brown, K. S., Myers, C. R., \& Sethna, J. P. (2007). Universally sloppy parameter sensitivities in systems biology models. PLoS computational biology, 3(10).

Haaf, J. M., \& Rouder, J. N. (2017). Developing constraint in bayesian mixed models. Psychological methods, 22(4), 779 .

Hoekstra, R., Morey, R. D., Rouder, J. N., \& Wagenmakers, E.-J. (2014). Robust misinterpretation of confidence intervals. Psychonomic bulletin \& review, 21(5), 1157-1164.

Holmes, W. R. (2015). A practical guide to the probability density approximation (pda) with improved implementation and error characterization. Journal of Mathematical Psychology, $68,13-24$.

Hübner, R., Steinhauser, M., \& Lehle, C. (2010). A dual-stage two-phase model of selective attention. Psychological Review, 117(3), 759.

Kass, R. E., \& Raftery, A. E. (1995). Bayes factors. Journal of the american statistical association, 90(430), 773-795.

Kruschke, J. K. (2013). Bayesian estimation supersedes the t test. Journal of Experimental Psychology: General, 142(2), 573.

Kruschke, J. K., \& Liddell, T. M. (2018). The bayesian new statistics: Hypothesis testing, estimation, meta-analysis, and power analysis from a bayesian perspective. Psychonomic Bulletin Es Review, 25(1), 178-206.

Lerche, V., \& Voss, A. (2016). Model complexity in diffusion modeling: Benefits of making the model more parsimonious. Frontiers in psychology, 7, 1324.

Lerche, V., \& Voss, A. (2017). Experimental validation of the diffusion model based on a slow 
response time paradigm. Psychological research, 1-16.

Lerche, V., Voss, A., \& Nagler, M. (2017). How many trials are required for parameter estimation in diffusion modeling? a comparison of different optimization criteria. Behavior Research Methods, 49(2), 513-537.

Lin, H., Saunders, B., Friese, M., Evans, N. J., \& Inzlicht, M. (2020). Strong effort manipulations reduce response caution: A preregistered reinvention of the ego-depletion paradigm. Psychological Science.

Pilly, P. K., \& Seitz, A. R. (2009). What a difference a parameter makes: A psychophysical comparison of random dot motion algorithms. Vision research, 49(13), 1599-1612.

Rae, B., Heathcote, A., Donkin, C., Averell, L., \& Brown, S. (2014). The hare and the tortoise: Emphasizing speed can change the evidence used to make decisions. Journal of Experimental Psychology: Learning, Memory, and Cognition, 40(5), 1226.

Ratcliff, R. (1978). A theory of memory retrieval. Psychological review, 85(2), 59.

Ratcliff, R., \& Rouder, J. N. (1998). Modeling response times for two-choice decisions. Psychological science, $9(5), 347-356$.

Ratcliff, R., Thapar, A., \& McKoon, G. (2001). The effects of aging on reaction time in a signal detection task. Psychology and aging, 16(2), 323.

Ratcliff, R., \& Tuerlinckx, F. (2002). Estimating parameters of the diffusion model: Approaches to dealing with contaminant reaction times and parameter variability. Psychonomic bulletin Es review, 9(3), 438-481.

Rouder, J. N., Morey, R. D., Speckman, P. L., \& Province, J. M. (2012). Default bayes factors for anova designs. Journal of Mathematical Psychology, 56 (5), 356-374.

Salthouse, T. A. (1996). The processing-speed theory of adult age differences in cognition. Psychological review, 103(3), 403.

Shadlen, M. N., \& Newsome, W. T. (1996). Motion perception: seeing and deciding. Proceedings of the national academy of sciences, 93(2), 628-633.

Starns, J. J., \& Ratcliff, R. (2012). Age-related differences in diffusion model boundary optimality with both trial-limited and time-limited tasks. Psychonomic bulletin $\&$ review, 19(1), 139145. 
Stone, M. (1960). Models for choice-reaction time. Psychometrika, 25(3), 251-260.

Ter Braak, C. J. (2006). A markov chain monte carlo version of the genetic algorithm differential evolution: easy bayesian computing for real parameter spaces. Statistics and Computing, $16(3), 239-249$.

Tillman, G., Strayer, D., Eidels, A., \& Heathcote, A. (2017). Modeling cognitive load effects of conversation between a passenger and driver. Attention, Perception, 83 Psychophysics, $79(6)$, $1795-1803$.

Turner, B. M., Sederberg, P. B., Brown, S. D., \& Steyvers, M. (2013). A method for efficiently sampling from distributions with correlated dimensions. Psychological methods, 18(3), 368.

Ulrich, R., Schröter, H., Leuthold, H., \& Birngruber, T. (2015). Automatic and controlled stimulus processing in conflict tasks: Superimposed diffusion processes and delta functions. Cognitive psychology, 78, 148-174.

van Ravenzwaaij, D., Dutilh, G., \& Wagenmakers, E.-J. (2012). A diffusion model decomposition of the effects of alcohol on perceptual decision making. Psychopharmacology, 219(4), 10171025.

van Ravenzwaaij, D., \& Oberauer, K. (2009). How to use the diffusion model: Parameter recovery of three methods: Ez, fast-dm, and dmat. Journal of Mathematical Psychology, 53(6), 463-473.

van Ravenzwaaij, D., \& Wagenmakers, E.-J. (2019). Advantages masquerading as issues in bayesian hypothesis testing: A commentary on tendeiro and kiers (2019). PsyArXiv.

Voss, A., Rothermund, K., \& Voss, J. (2004). Interpreting the parameters of the diffusion model: An empirical validation. Memory \&3 cognition, 32(7), 1206-1220.

Voss, A., \& Voss, J. (2007). Fast-dm: A free program for efficient diffusion model analysis. Behavior Research Methods, 39(4), 767-775.

Wagenmakers, E.-J. (2007). A practical solution to the pervasive problems of p values. Psychonomic Bulletin $\&$ Review, 14(5), 779-804.

Wagenmakers, E.-J., \& Farrell, S. (2004). Aic model selection using akaike weights. Psychonomic bulletin $\&$ review, 11(1), 192-196.

Wagenmakers, E.-J., Ratcliff, R., Gomez, P., \& McKoon, G. (2008). A diffusion model account of criterion shifts in the lexical decision task. Journal of memory and language, 58(1), 140-159. 
Webster, D. M., \& Kruglanski, A. W. (1994). Individual differences in need for cognitive closure. Journal of personality and social psychology, 67(6), 1049.

White, C. N., Ratcliff, R., \& Starns, J. J. (2011). Diffusion models of the flanker task: Discrete versus gradual attentional selection. Cognitive psychology, 63(4), 210-238. 

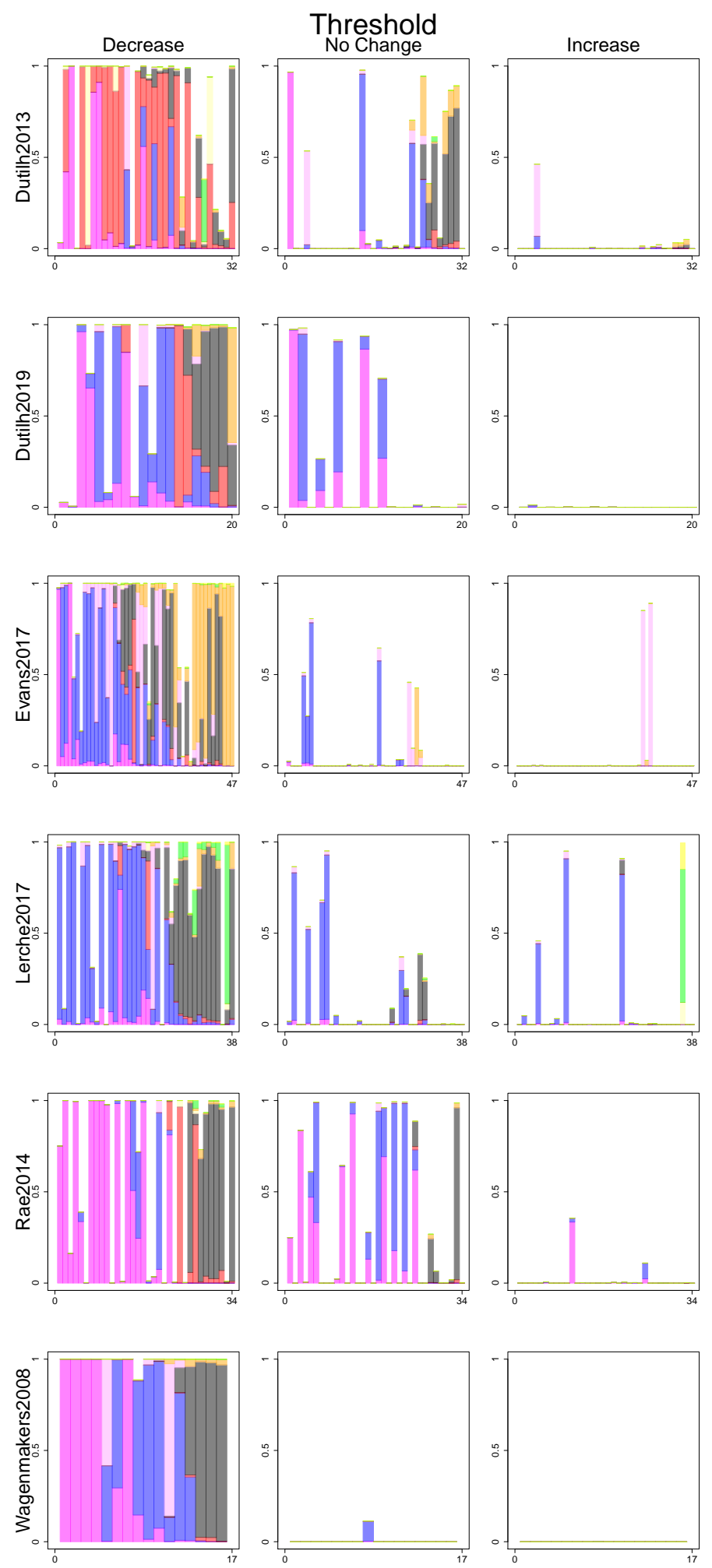

Figure 1. Displays the probability weights assigned to each model for the diffusion model analysis. A detailed description of the plotting format can be seen in the "Analysis framework" section of the main text. 

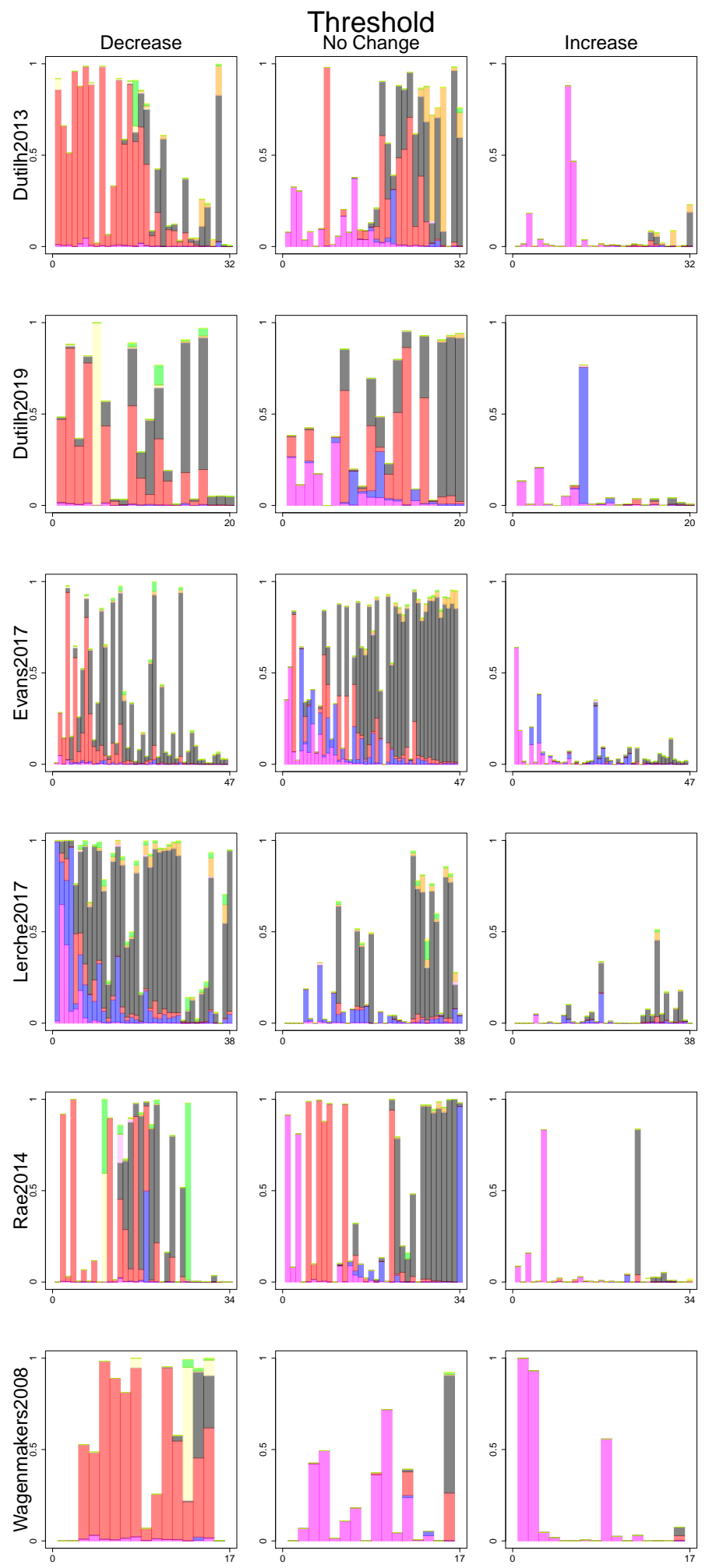

Figure 2. Displays the probability weights assigned to each model for the LBA analysis. A detailed description of the plotting format can be seen in the "Analysis framework" section of the main text. 

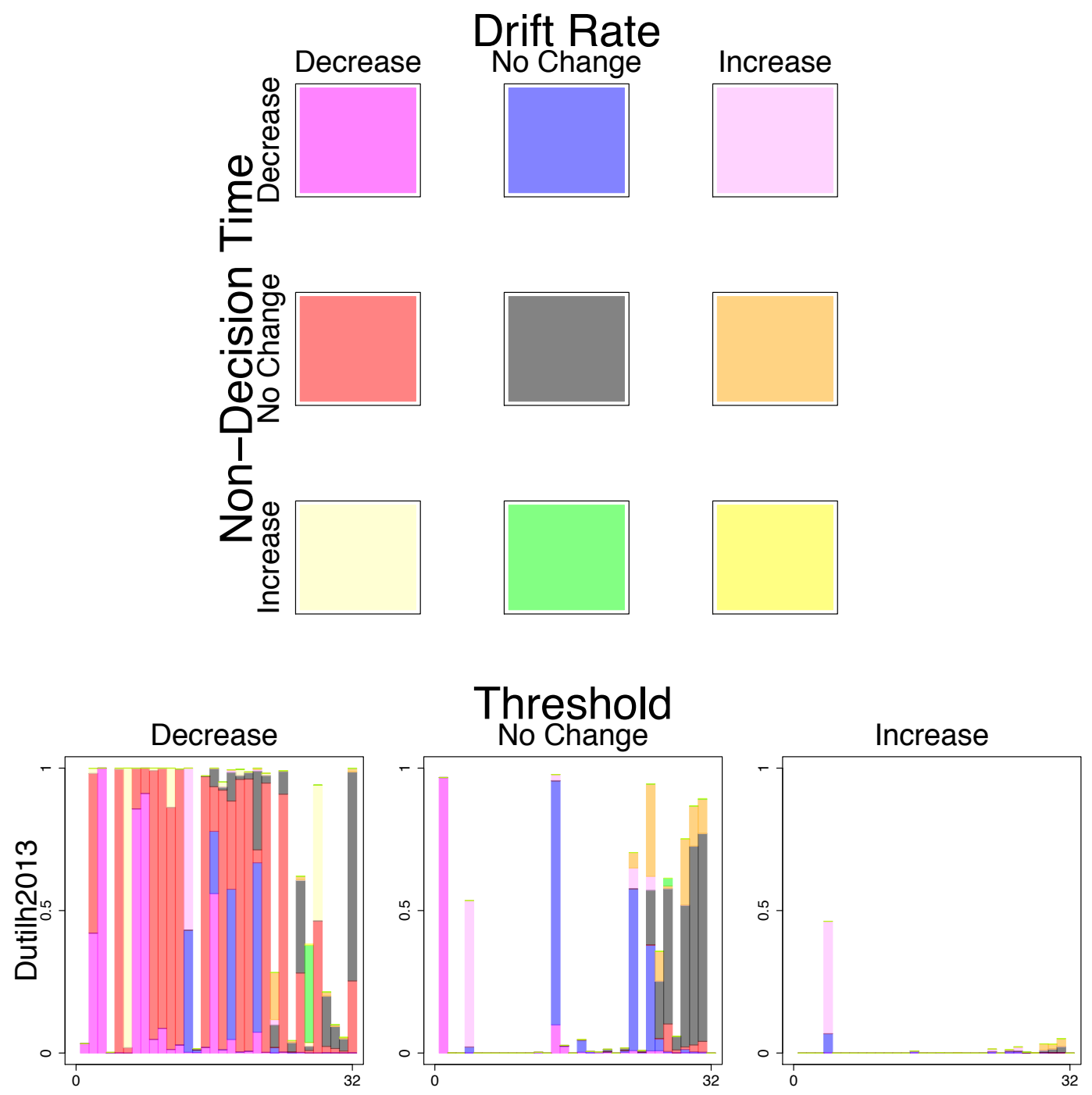

Figure 3. An example of the probability weights figures (Figures 1 and 2) using the diffusion model analysis of Dutilh et al. (2013). 

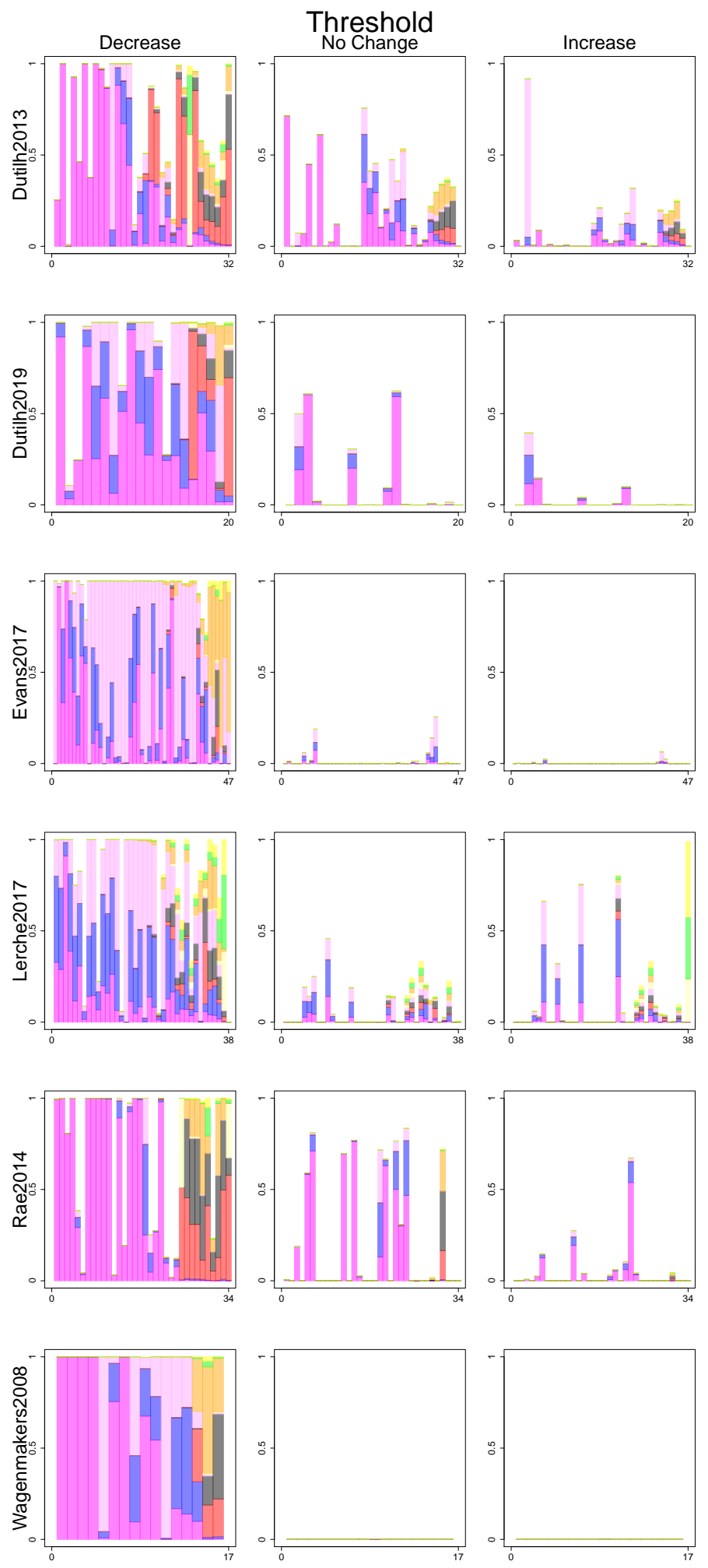

Figure 4. Displays the probability weights assigned to each model for the diffusion model analysis using tighter priors. A detailed description of the plotting format can be seen in the "Analysis framework" section of the main text. 

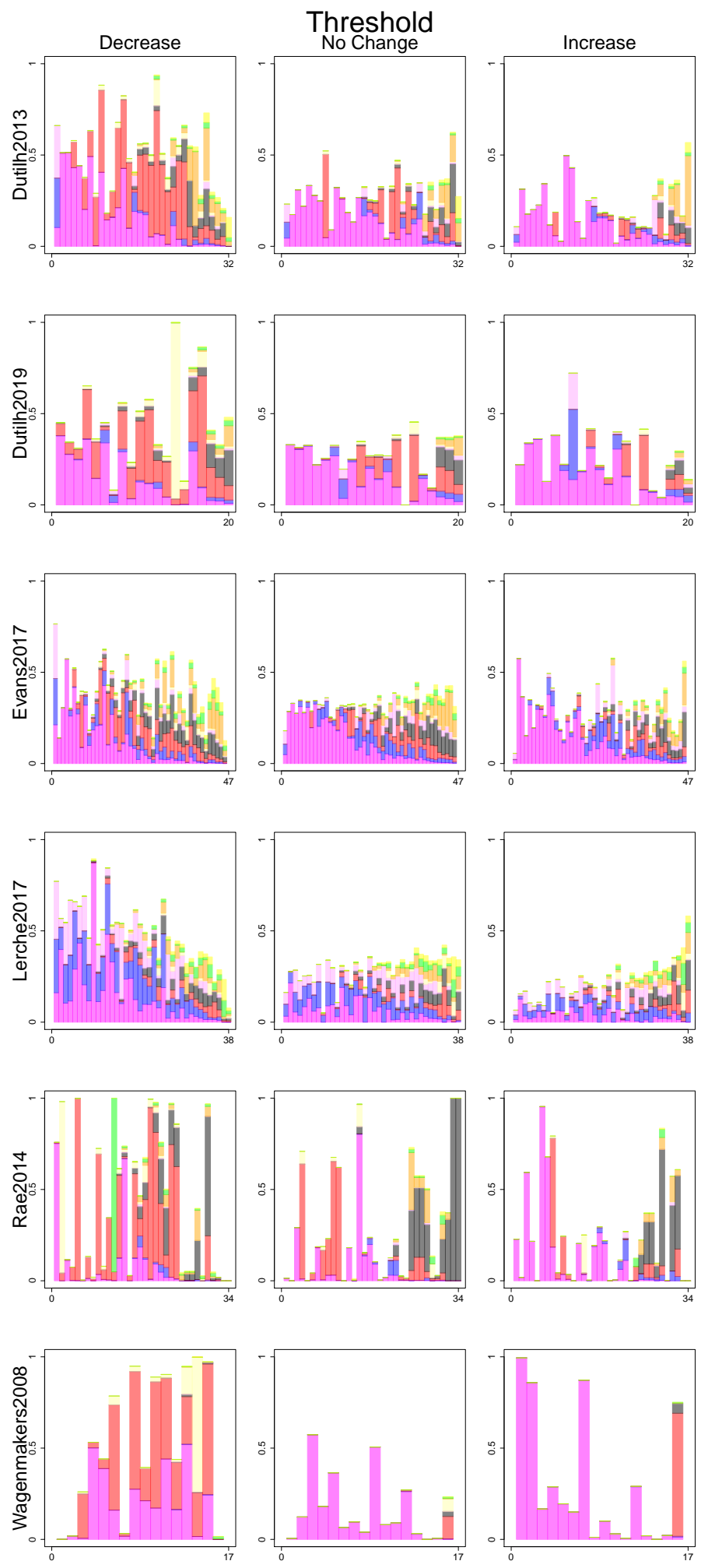

Figure 5. Displays the probability weights assigned to each model for the LBA analysis using tighter priors. A detailed description of the plotting format can be seen in the "Analysis framework" section of the main text. 